\title{
Apigenin-induced apoptosis of leukemia cells is mediated by a bimodal and differentially regulated residue-specific phosphorylation of heat-shock protein-27
}

\author{
ME Gonzalez-Mejia ${ }^{1,2}$, OH Voss ${ }^{1,2}$, EJ Murnan ${ }^{1}$ and AI Doseff ${ }^{*}, 1$
}

\begin{abstract}
Apigenin, a natural plant flavonoid with antiproliferative activity, is emerging as a promising compound for cancer prevention and therapy, but its mechanism of action remains unclear. High expression of the small heat-shock protein-27 (Hsp27) in leukemia contributes to the resistance of these cells to cancer treatments. Changes in Hsp27 phosphorylation have been associated with heat and metabolic stress, but its role in flavonoid anticancer activity has not been investigated. In this study, we examined the effect of apigenin in the regulation of Hsp27 on leukemia. We showed that apigenin does not affect Hsp27 expression but induces a bimodal phosphorylation on Ser78 and Ser82. The phosphorylation at early times was regulated by p38. At later times, Hsp27 phosphorylation was dependent on p38 activity and for some residues on PKC $\delta$. Silencing of p38 expression reduced apigenin-induced phosphorylation on Ser15, Ser78, and Ser82, whereas silencing of PKC $\delta$ expression reduced the phosphorylation on Ser15 and Ser82 without affecting Ser78. In addition, we found that apigenin-induced PKC $\delta$ activity is mediated by $\mathrm{p38}$. We also showed that the phosphorylation of Hsp27 significantly increased the susceptibility of leukemia cells to apigenin-induced apoptosis. Together, these results identify a complex signaling network regulating the cytotoxic effect of apigenin through Hsp27 phosphorylation.
\end{abstract}

Cell Death and Disease (2010) 1, e64; doi:10.1038/cddis.2010.41; published online 19 August 2010

Subject Category: Cancer

Flavonoids have long been recognized as having potential anticancer, anti-inflammatory, antioxidant, and antimicrobial properties, serving as important nutraceutical components of our diet. ${ }^{1-4}$ Apigenin (4',5,7-trihydroxyflavone), a common plant dietary flavonoid found at high levels in parsley and celery, abundant vegetables in the Mediterranean's diet, ${ }^{5}$ is emerging as an alternative anticancer compound, but its mechanism of action remains unclear. Apigenin induces apoptosis with effectiveness in leukemia cells but to a lesser extent on other cancer cells. ${ }^{6-10}$ Moreover, apigenin showed no antiproliferative effect in normal cells, suggesting its potential relevance as an anticancer compound. ${ }^{9}$

Heat-shock proteins-27 (Hsp27) belongs to the conserved family of small heat-shock protein chaperones. ${ }^{11}$ Hsp27 associates with components of the extrinsic and intrinsic apoptotic pathways, inhibiting the execution of the apoptosis and is emerging as an antiapoptotic factor. ${ }^{12-15}$ Elevated expression of Hsp27 contributes to an increase in tumorigenicity and resistance to chemotherapy characteristic of malignant cells. ${ }^{16}$ In leukemia, high Hsp27 expression is associated with adverse prognosis. ${ }^{17}$ Changes on Hsp27 phosphorylation have been associated with heat and oxidative stresses. ${ }^{11,18-20}$ Phosphorylation of Hsp27 modulates its oligomerization properties, thus probably modulating Hsp27-cytoprotective activity. ${ }^{21-23} \mathrm{Hsp} 27$ is most commonly phosphorylated in vivo as a result of stress conditions on Ser15, Ser78, and Ser82 (referred in the text as S15, S78, and $S 82,{ }^{24,25}$ ). Aberrant Hsp27 phosphorylation has been linked to several clinical conditions. Hsp27 is phosphorylated mainly through mitogen-activated protein kinase-activated protein kinases (MKK2/3) as a result of the activation of the mitogen-activated protein kinase p38 (p38). ${ }^{24,25}$ In addition, protein kinase $\mathrm{C} \delta(\mathrm{PKC} \delta)$ can phosphorylate Hsp27.26,27 Also, the extracellular signal-regulated kinases (ERK1/2, referred as ERK) became activated through $\mathrm{PKC} \delta$ regulating MKK2/3 activity. ${ }^{28,29}$ However, the effect of dietary apigenin on Hsp27 has not been addressed.

In this study, we investigated the regulation of Hsp27 by apigenin in leukemia cells. We found a bimodal phosphorylation of Hsp27 induced by apigenin. Using pharmacological

\footnotetext{
${ }^{1}$ Department of Molecular Genetics, Department Internal Medicine, Division of Pulmonary and Critical Care, The Heart and Lung Research Institute, The Ohio State University, Columbus, $\mathrm{OH} 43210$, USA

*Corresponding author: AI Doseff, Internal Medicine/Molecular Genetics, The Ohio State University, 201 Heart and Lung Research Institute, 473 West 12th Avenue, Columbus, OH 43210, USA.

Tel: + 614292 9507; Fax: + 614293 4799; E-mail: doseff.1@osu.edu

${ }^{2}$ These authors contributed equally to this work.

Keywords: flavonoids; apigenin; Hsp27; phosphorylation; apoptosis; $\mathrm{PKC} \delta$; 338

Abbreviations: ERK, extracellular signal-regulated kinases; Hsp27, heat-shock proteins-27; PKC $\delta$, protein kinase $\mathrm{C} \delta$; MKK, mitogen-activated protein kinaseactivated protein kinases

Received 08.3.10; revised 14.6.10; accepted 22.6.10; Edited by R De Maria
} 
inhibitors and siRNA, we found that apigenin induces an 'early' p38-dependent phosphorylation on S78 and S82. A 'late' phosphorylation on S15, S78, and S82 is regulated by notably different mechanisms. $\mathrm{S} 15$ and $\mathrm{S} 82$ phosphorylation is regulated in a $\mathrm{p38} / \mathrm{PKC} \delta$ manner. However, phosphorylation on $\mathrm{S} 78$ is regulated by $\mathrm{p} 38$ but is $\operatorname{PKC} \delta$ independent. In addition, both 'early' and 'late' apigenin-induced Hsp27 phosphorylation events are ERK independent. We determined, using phosphor-mimic mutants, that Hsp27 phosphorylation significantly increased the susceptibility of leukemia cells to apigenin-induced apoptosis. These findings establish that apigenin induces changes in Hsp27 phosphorylation contributing to the cytotoxic activity of the flavonoid.

\section{Results}

Apigenin increases Hsp27 phosphorylation. Apigenin induces apoptosis in leukemia cells more effectively than in other cells including, among others, lung or breast epithelial cells and has no effect in normal fibroblast, but the basis of this difference remains unknown. ${ }^{9}$ We previously showed that Hsp27 is highly and constitutively expressed in monocytic leukemia cells. ${ }^{15}$ Moreover, high expression of Hsp27 has been correlated with chemoresistance. To determine the effect of apigenin in Hsp27, THP-1 cells were treated with $50 \mu \mathrm{M}$ apigenin, an amount shown to induce apoptosis. ${ }^{9} \mathrm{We}$ found that apigenin had no effect on the total expression level of Hsp27 (Figure 1a, Hsp27). However, western blot analysis using antibodies that recognize specifically Hsp27phosphorylated residues S15, S78, or S82 (Hsp27-pS15, Hsp27-pS78, and Hsp27-pS82, respectively) showed that apigenin induced a rapid and transient phosphorylation on S78 and S82 after $15 \mathrm{~min}$, referred through out the text as 'early' phosphorylation (Figure 1a). This phosphorylation was followed by a later increase observed at $6 \mathrm{~h}$ on S15, S78, and S82, named 'late' phosphorylation (Figure 1a).

As phosphorylation of $\mathrm{Hsp} 27$ is regulated during heat shock by MAPKs, we next investigated the effect of apigenin on p38 and ERK. We found that after $15 \mathrm{~min}$ apigenin induced the transient phosphorylation of p38, followed by a second increase in phosphorylation at $6 \mathrm{~h}$ that remained high even at $9 \mathrm{~h}$ (Figure 1b. p-p38). In addition, ERK phosphorylation increased after $3 \mathrm{~h}$, reaching its maximum at approximately $6 \mathrm{~h}$ (Figure 1b, p-ERK).

As Hsp27 phosphorylation can be regulated by $\mathrm{PKC} \delta$ in other stress conditions, we next determined the effect of apigenin on the activity of $\operatorname{PKC} \delta$. For this purpose, lysates from THP-1 cells treated with $50 \mu \mathrm{M}$ apigenin for different times were immunoprecipitated with anti-PKC $\delta$ antibodies, or with an isotype control, and the immunoprecipitates were subjected to in vitro kinase assays using histone $2 \mathrm{~B}(\mathrm{H} 2 \mathrm{~B})$ as exogenous substrate. We found that $\mathrm{PKC} \delta$ activity increased at $6 \mathrm{~h}$ (Figure 1c, H2B).

These results show that apigenin had no effect on the expression level of Hsp27 but did induced changes in Hsp27 phosphorylation. Apigenin induced a rapid and steady activation of $\mathrm{p} 38$ that coincides with the 'early' phosphorylation of Hsp27 and a 'late' phosphorylation of Hsp27 concurrent to the activation of ERK and $\mathrm{PKC} \delta$.
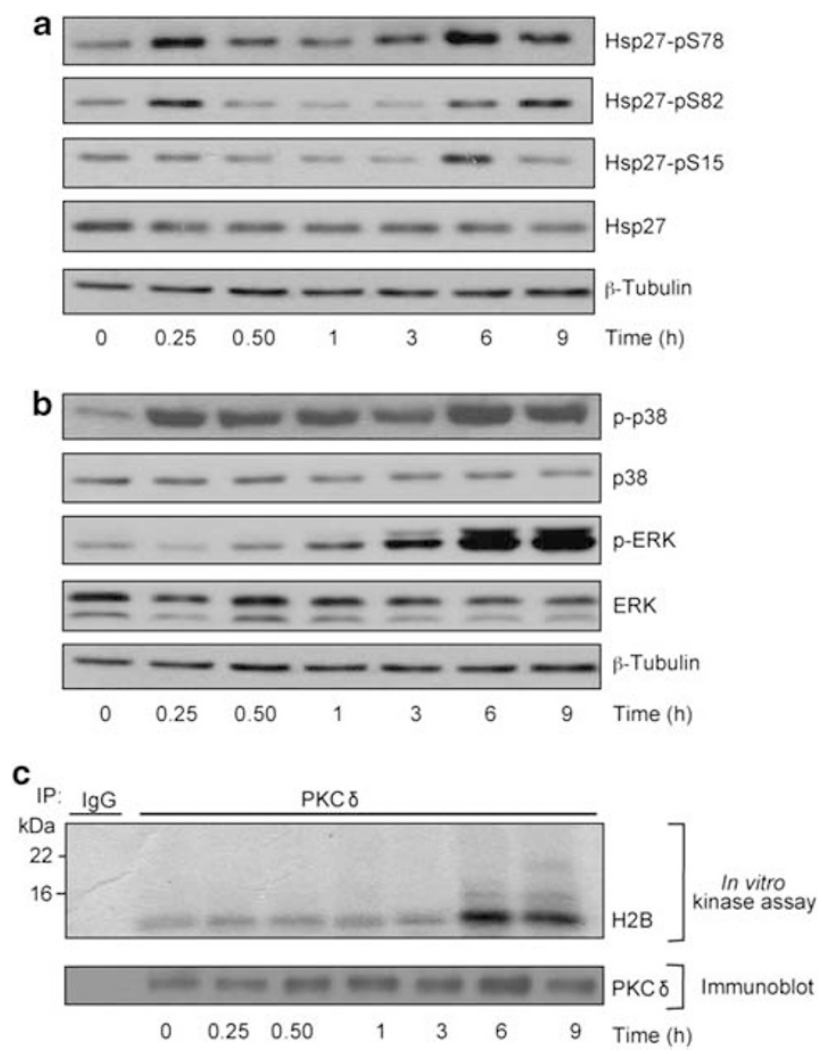

Figure 1 Apigenin induces phosphorylation of Hsp27 and activation of p38 and ERK-MAPK and PKC $\delta$. Lysates from THP- 1 cells treated with $50 \mu \mathrm{M}$ apigenin for different lengths of time were analyzed by immunobloting with (a) anti-phosphoHsp27-pS78, anti-phospho-Hsp27-pS82, anti-phospho-Hsp27-pS15, anti-totalHsp27, and anti-Tubulin antibodies. (b) Immunoblots with anti-phospho-p38, antiphospho-ERK, anti-total-p38, anti-total-ERK, and anti- $\beta$-Tubulin antibodies. (c) PKC $\delta$ kinase activity was determined by in vitro kinase assays. Total cell lysates were immunoprecipitated (IP) with anti-PKC $\delta$ antibodies or an isotype control (IgG) followed by in vitro kinase assay in the presence of $\left(\gamma^{32} \mathrm{P}\right)$ ATP and H2B as substrate. Phosphorylated kinase products were resolved by SDS-PAGE and transferred to a nitrocellulose membrane. Phospholabeled proteins were visualized by autoradiography. Immunoblots of the same membrane with PKC $\delta$ served as loading control. Results are a representative of four independent experiments

Differential regulation of early and late Hsp27 phosphorylation. Because apigenin induced the activation of p38 at $15 \mathrm{~min}$, a time that coincides with the 'early' phosphorylation of Hsp27, we investigated whether p38 was involved in the regulation of Hsp27 phosphorylation in apigenin-treated cells. THP-1 cells were pretreated for $1 \mathrm{~h}$ with the p38 inhibitor SB203580 or with diluent $(-)$ before the addition of apigenin for $15 \mathrm{~min}$. We found that the 'early' phosphorylation of $\mathrm{Hsp} 27$ at $\mathrm{S} 78$ and $\mathrm{S} 82$ induced by apigenin was inhibited in cells pretreated with the p38 inhibitor (Figure 2, lane 3 versus 2, Hsp27-pS78 and Hsp27-pS82). The 'early' phosphorylation observed was induced by apigenin, as cells treated with diluent for $15 \mathrm{~min}$ or the p38 inhibitor and diluent showed just a basal signal (Figure 2, lanes 1 and 4, respectively). These results suggest that p38 regulates the 'early' phosphorylation of Hsp27 at both $\mathrm{S} 78$ and $\mathrm{S} 82$.

To delineate the signaling pathway involved in the 'late' phosphorylation of Hsp27, we first studied the effect of ERK. 


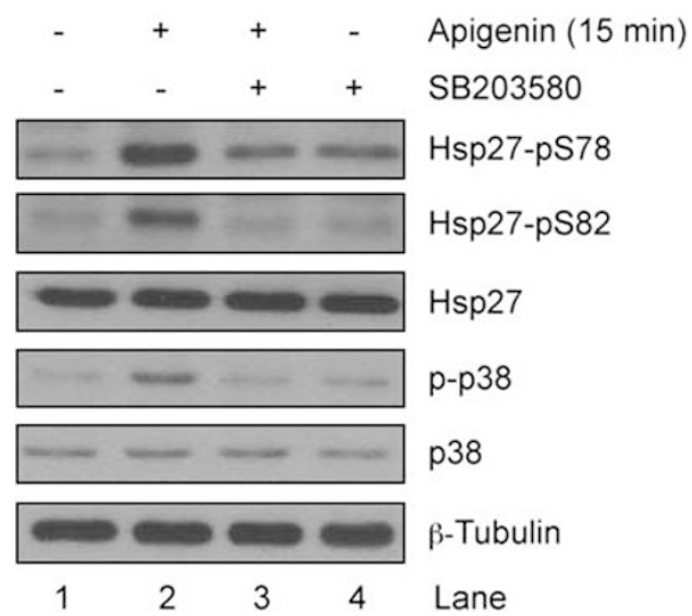

Figure 2 Apigenin-induced 'early' Hsp27 phosphorylation is dependent on p38 activity. Lysates from THP-1 cells treated with the diluent alone (lane 1), pretreated with $10 \mu \mathrm{M}$ p38 inhibitor SB203580 or diluent (lanes 3 and 2) before the addition of $50 \mu \mathrm{M}$ apigenin for $15 \mathrm{~min}$ following or treated with the p38 inhibitor SB203580, and diluent (lane 4) were immunoblotted with anti-phospho-Hsp27-pS78, antiphospho-Hsp27-pS82, anti-phospho-p38, anti-total-Hsp27, anti-total-p38 antibodies. The same membrane was immunoblotted with anti-Tubulin antibodies as loading control. Results are a representative of four independent experiments

THP-1 cells were pretreated with diluent control or the ERK inhibitor PD98059 for $1 \mathrm{~h}$ before the addition of $50 \mu \mathrm{M}$ apigenin. Treatment with the ERK inhibitor had no effect on apigenin-induced phosphorylation of Hsp27 (Figure 3, lane 3 versus 2 ), and cells treated with the inhibitor or diluent alone showed no phosphorylation of Hsp27 (Figure 3, lane 4 and 1). In addition, the presence of the ERK inhibitor had no effect on the phosphorylation of p38 induced by apigenin but inhibited, as expected, the phosphorylation of ERK (Figure 3).

Next, we investigated the role of p38 and $\operatorname{PKC} \delta$ on the 'late' Hsp27 phosphorylation using pharmacological inhibitors. THP-1 cells were pretreated with the p38 inhibitor SB203580, or the PKC $\delta$ inhibitor rottlerin, or both inhibitors, or with diluent for $1 \mathrm{~h}$ before the addition of $50 \mu \mathrm{M}$ apigenin for $6 \mathrm{~h}$. We found that apigenin-induced phosphorylation of S78 was significantly inhibited in the presence of the p38 inhibitor SB203580 (Figure 4a, lane 3 versus $2,{ }^{\star \star} P<0.001$ ), reaching levels similar to that in control cells (Figure 4a, lane 3 versus 1 ). In contrast, the $\mathrm{PKC} \delta$ inhibitor rottlerin had no effect on the phosphorylation of S78 (Figure $4 a$, lane 4 versus 2 , NS = nonstatistically significant). Addition of both SB203580 and rottlerin showed decreased $S 78$ phosphorylation to levels similarly observed in the presence of the p38 inhibitor alone (Figure 4a, lane 5 versus 3 ). However, we found no additive or synergistic effects when cells were treated with both inhibitors simultaneously (Figure $4 a$, lane 5 versus $2,{ }^{\star \star} P<0.001$ ). The phosphorylation of S82 induced by apigenin was significantly inhibited by pretreatment with the p38 inhibitor SB203580 (Figure $4 \mathrm{~b}$, lane 3 versus $2,{ }^{\star} P<0.05$ ). In contrast to the lack of effect observed on $\mathrm{S} 78$, the $\mathrm{PKC} \delta$ inhibitor rottlerin, significantly inhibited apigenin-induced phosphorylation of S82 (Figure $4 \mathrm{~b}$, lane 4 versus $2,{ }^{\star} P<0.05$ ). Similar to S82 phosphorylation, we observed that the pretreatment with the p38 inhibitor SB203580 inhibited apigenin-induced phosphorylation of $\mathrm{S} 15$ (Figure $4 \mathrm{c}$, lane 3 versus $2,{ }^{\star} P<0.05$ ).

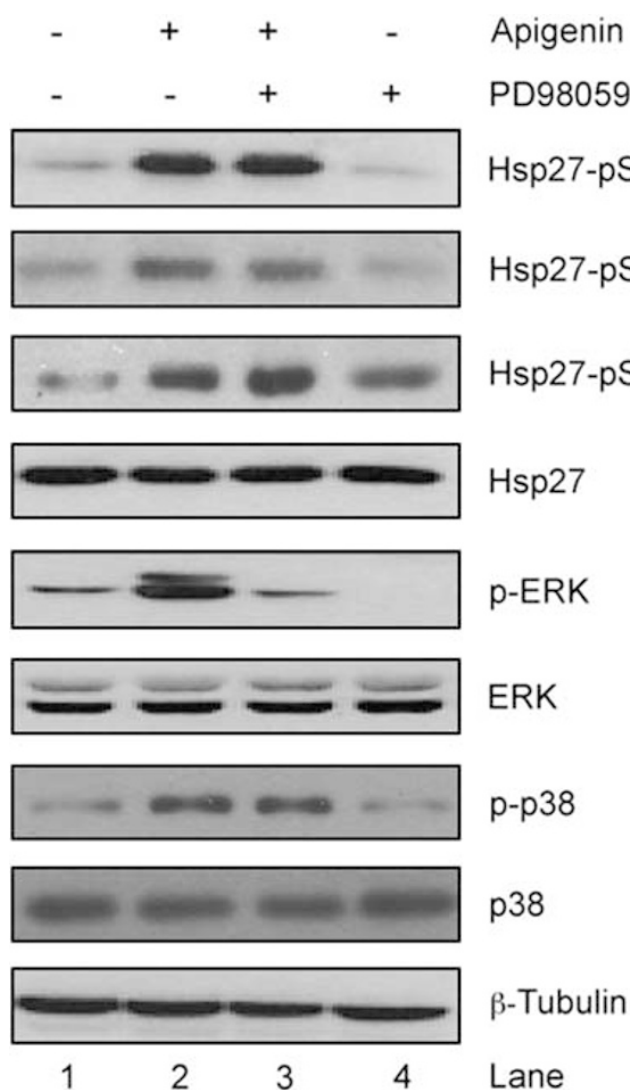

Figure 3 Apigenin-induced 'late' Hsp27 phosphorylation is ERK-independent. Lysates from THP-1 cells were treated with diluent (lane 1), or pretreated with diluent or $25 \mu \mathrm{M}$ PD98059 before the addition of $50 \mu \mathrm{M}$ apigenin for $6 \mathrm{~h}$ (lanes 2 and 3 , respectively) and immunoblotted with anti-phospho-Hsp27-pS78, anti-phosphoHsp27-pS82, anti-phospho-Hsp27-pS15, anti-phospho-ERK, anti-total-Hsp27, anti-total-ERK antibodies. The same membrane was immunoblotted with anti$\beta$-Tubulin antibodies as loading control. Results are a representative of four independent experiments

In addition, rottlerin decreased apigenin-induced phosphorylation of $\mathrm{S} 15$ (Figure $4 \mathrm{c}$, lane 4 versus $2,{ }^{\star} P<0.05$ ). The addition of both inhibitors, however, showed no additive or synergistic effects (Figure 4c, lanes 5, 4 versus 2, $\left.{ }^{\star *} P<0.001\right)$.

To further study the signaling network responsible for apigenin-induced Hsp27 phosphorylation and to take into consideration the possible limitation of specificity of kinase inhibitors, we next assessed the effect of silencing p38 and $\mathrm{PKC} \delta$, respectively. THP-1 cells were transfected with siRNAp38 or siRNA-Control, and the phosphorylation status of Hsp27 was evaluated after treatment with $50 \mu \mathrm{M}$ of apigenin or diluent for $6 \mathrm{~h}$. We found that silencing of $\mathrm{p} 38$ significantly decreased apigenin-induced phosphorylation of S15, S78, and S82 (Figure 5a, lane 6 versus 4), whereas apigenininduced phosphorylation of Hsp27 remained similarly elevated in cells transfected with siRNA-Control or in nontransfected (Figure 5a, lane 4 versus 2). Silencing of PKC $\delta$ decreased phosphorylation of S15 and S82, but had no effect on apigenin-induced phosphorylation of $S 78$ (Figure $5 \mathrm{~b}$, lane 6 versus 4). These results suggest that the 'late' phosphorylation of Hsp27 is regulated by different signaling networks 

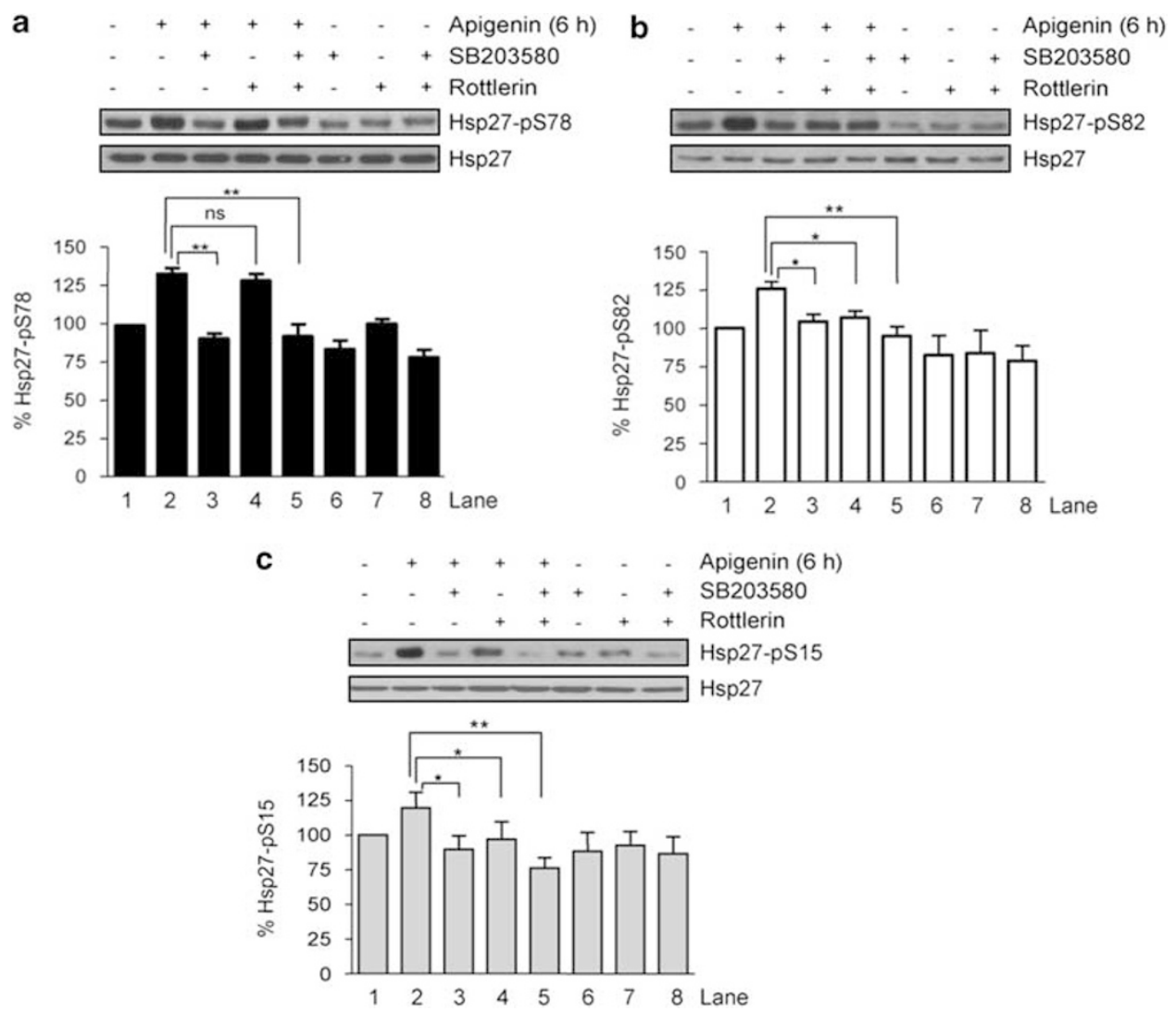

Figure 4 'Late' Hsp27 phosphorylation induced by apigenin is modulated by p38-MAPK and PKC $\delta$. Lysates from THP-1 cells treated with $50 \mu \mathrm{M}$ apigenin for $6 \mathrm{~h}$ and

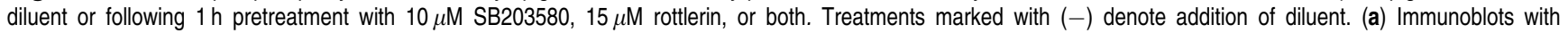
anti-phospho-Hsp27-pS78 and anti-total-Hsp27 antibodies. (b) Immunoblots with anti-phospho-Hsp27-pS82 and anti-total-Hsp27 antibodies. (c) Immunoblots with antiphospho-Hsp27-pS15 and anti-total-Hsp27 antibodies. Densitometry data normalized by the loading control are represented as mean \pm S.E.M. $\left(N=5\right.$, ${ }^{\star} P \leqslant 0.05$ and ${ }^{* *} P \leqslant 0.001$, NS denotes non-statistical significance)

depending on the amino-acid residue; $\mathrm{S} 78$ is regulated by p38 while phosphorylation of $\mathrm{S} 15$ and $\mathrm{S} 82$ is mediated by both p38 and $\mathrm{PKC} \delta$.

PKC $\delta$ activity is modulated by p38. As both $\mathrm{PKC} \delta$ and $\mathrm{p} 38$ regulate the 'late' phosphorylation of Hsp27, we next studied the relationship of these kinases in the pathway. As shown in Figure $6 \mathrm{a}$, cells treated with apigenin for $6 \mathrm{~h}$ have elevated levels of phosphorylated p38 when compared with cells treated with diluent (Figure 6a, lane 2 versus 1 ). We found that pretreatment with the $\mathrm{PKC} \delta$ inhibitor rottlerin before the addition of apigenin had no effect on the phosphorylation status of p38 (Figure 6a, lane 4 versus 2), whereas SB203580 inhibited apigenin-induced phosphorylation of p38 (Figure 6a, lane 3 versus 2). Next, we determined the effect of inhibiting p38 on the apigenin-induced activity of $\mathrm{PKC} \delta$. For this purpose, lysates from THP-1 cells pretreated with the p38 inhibitor SB203580 or diluent and followed by $6 \mathrm{~h}$ of treatment with $50 \mu \mathrm{M}$ apigenin were immunoprecipitated with anti-PKC $\delta$ antibodies. Immunoprecipitates were subjected to in vitro kinase assays using $\mathrm{H} 2 \mathrm{~B}$ as exogenous substrate. We found that apigenin-induced $\operatorname{PKC} \delta$ activity was decreased in cells treated with the p38 inhibitor (Figure 6b, lane 4 versus 3 ). This effect was similar to the inhibition observed in cells treated with apigenin in the presence of the
$\mathrm{PKC} \delta$ inhibitor rottlerin (Figure 6b, lane 5 versus 4). A similar decrease in $\mathrm{PKC} \delta$ activity was found in cells treated with apigenin in the presence of both SB203580 and rottlerin (Figure 6b, lane 6 versus lanes 4 and 5).

To further evaluate whether the activity of $\mathrm{PKC} \delta$ was dependent on $\mathrm{p} 38$, we assessed $\mathrm{PKC} \delta$ activity in THP-1 cells transfected with siRNA-p38 or siRNA-Control. Lysates from cells transfected with siRNA-p38 or siRNA-Control and treated with $50 \mu \mathrm{M}$ apigenin for $6 \mathrm{~h}$ were immunoprecipitated with anti-PKC $\delta$ antibodies or an isotype control. Immunoprecipitates were subjected to in vitro kinase assays in the presence of $\mathrm{H} 2 \mathrm{~B}$ as exogenous substrate. We found reduced $\mathrm{PKC} \delta$ activity in siRNA-p38 lysates (Figure 6c, lane 2 versus 1). These results suggest that $\mathrm{p} 38$ regulates $\mathrm{PKC} \delta$ in the signal cascade induced by apigenin.

Phosphorylation of Hsp27 modulates apigenin-induced apoptosis. Phosphorylation of Hsp27 has been shown to modulate its protective activity against stress conditions. ${ }^{23}$ To assess whether the phosphorylation of Hsp27 affects apigenin-induced apoptosis, we examined the effect of phosphor-mimic mutants, in which all three S15, S78, and S82 were mutated to aspartic acids, Hsp27-D, or to alanines, Hsp27-A. ${ }^{23}$ Lysates from THP-1 cells transfected with an empty vector or either Hsp27 wild-type (Hsp27-WT), 


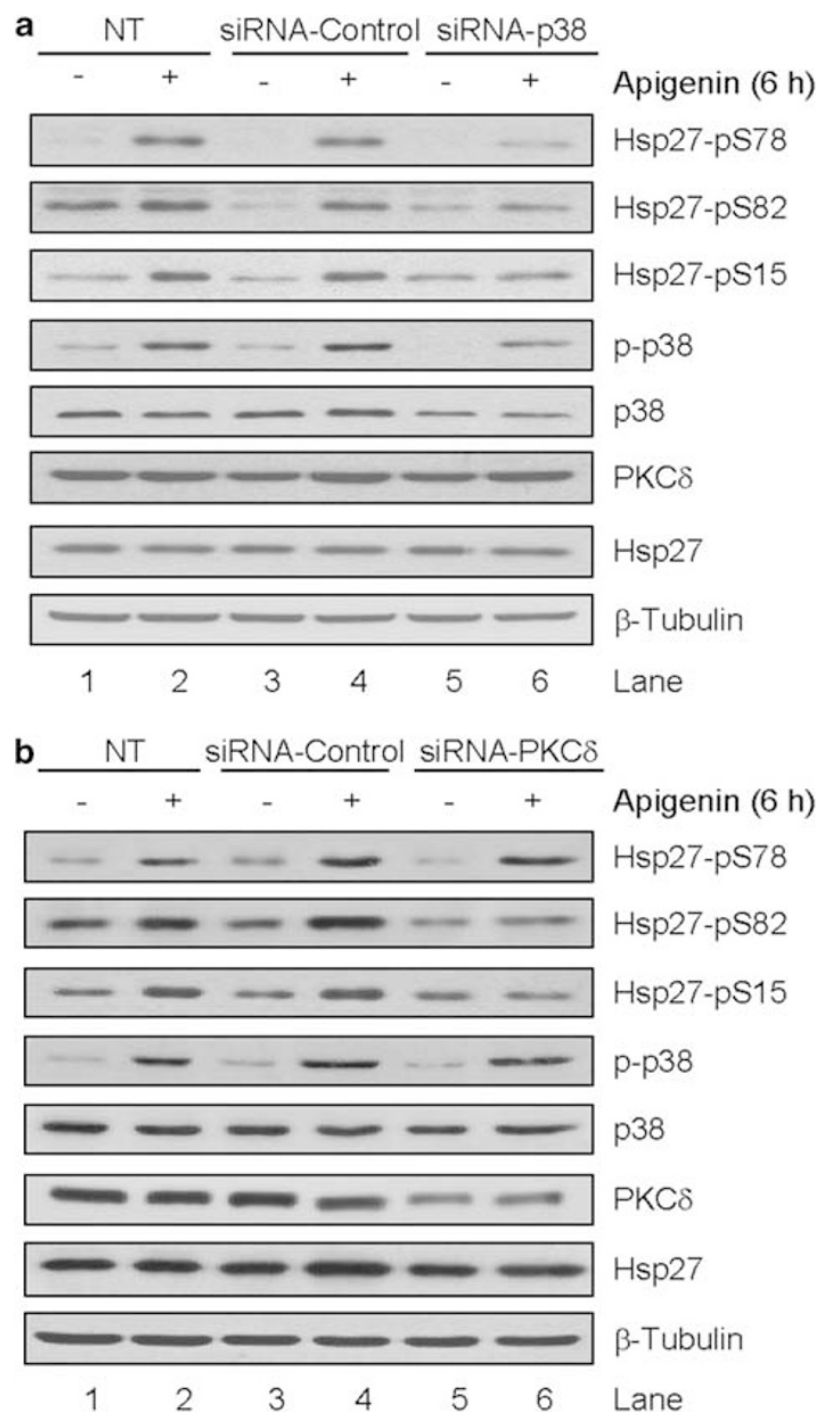

Figure $5 \mathrm{PKC} \delta$ and p38-MAPK regulate the 'late' phosphorylation of Hsp27 induced by apigenin. Silencing of p38 and PKC $\delta$ inhibits apigenin-induced-Hsp27 phosphorylation. (a) Immunoblots of lysates from THP-1 cells non-transfected (NT) or transfected with siRNA-Control or siRNA-p38 were treated with diluent $(-)$ or treated for $6 \mathrm{~h}$ with $50 \mu \mathrm{M}$ apigenin $(+)$. (b) Immunoblots of lysates from THP-1 cells non-transfected (NT) or transfected with siRNA-Control or siRNA-PKC $\delta$ and treated with diluent $(-)$ or treated for $6 \mathrm{~h}$ with $50 \mu \mathrm{M}$ apigenin $(+)$. Results are a representative of three independent experiments

Hsp27-A, or Hsp27-D were used to assess the expression of Hsp27-WT and the Hsp27 phosphor-mutants. Immunoblot analysis using the anti-Myc antibodies showed equal expression of Hsp27-WT, Hsp27-A, and Hsp27-D mutants (Figure 7a). Next, the cells were treated with diluent for $9 \mathrm{~h}$ (referred as NT) or treated with $50 \mu \mathrm{M}$ apigenin for 6 or $9 \mathrm{~h}$, times when Hsp27 phosphorylation was observed. In cells transfected with the empty vector, we found that apigenin induced $20 \%$ apoptosis by $9 \mathrm{~h}$ as determined by AnnexinV/7AAD staining (Figure 7a). This percentage of apoptosis was similar to numbers reported in non-transfected cells treated for $9 \mathrm{~h}$ with apigenin. ${ }^{9}$ Overexpression of Hsp27-WT reduced by half the percentage of cells undergoing apoptosis at $9 \mathrm{~h}$ (Figure 7a). Similar results were obtained in cells expressing

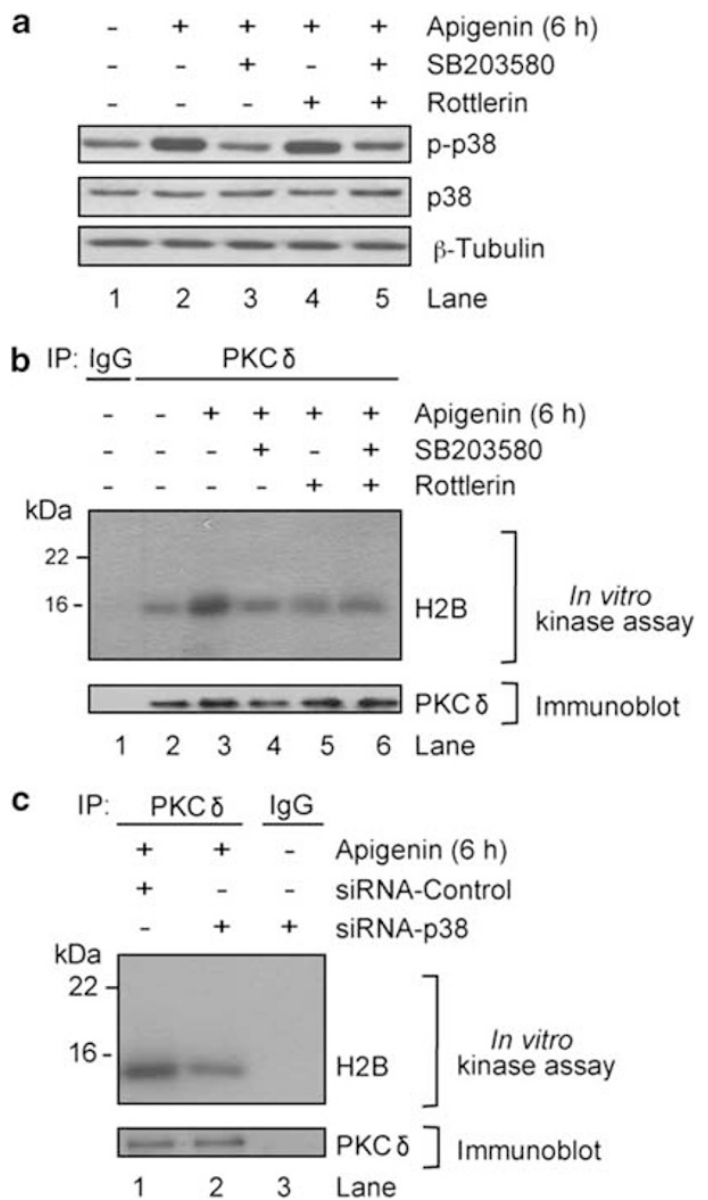

Figure 6 p38-MAPK modulates apigenin-induced PKC $\delta$ activity. THP-1 cells treated with $50 \mu \mathrm{M}$ apigenin for $6 \mathrm{~h}$ alone or following $1 \mathrm{~h}$ pretreatment with $10 \mu \mathrm{M}$ SB203580, a p38 inhibitor, $15 \mu \mathrm{M}$ rottlerin, a $\mathrm{PKC} \delta$ inhibitor, or both, and lanes marked with $(-)$, denote addition of diluent, were (a) immunobloted with antiphospho-p38, anti-total-p38, and anti- $\beta$-Tubulin antibodies. (b) Lysates were immunoprecipitated (IP) with anti-PKC $\delta$ antibodies or an isotype control ( $\operatorname{lgG})$, followed by in vitro kinase assay in the presence of $\left(\gamma^{32} \mathrm{P}\right) \mathrm{ATP}$ and $\mathrm{H} 2 \mathrm{~B}$ as a substrate. Kinase products were resolved by SDS-PAGE and transferred to a nitrocellulose membrane. Phospholabeled proteins were visualized by autoradiography. The same membrane was immunoblotted with anti-PKC $\delta$ antibodies as loading control. (c) Lysates from THP-1 cells transfected with siRNA-Control or siRNA-p38 were treated for $6 \mathrm{~h}$ with $50 \mu \mathrm{M}$ apigenin and immunoprecipitated (IP) with anti-PKC $\delta$ antibodies or an isotype control (IgG), followed by in vitro kinase assay in the presence of $\left(\gamma_{-}{ }^{32} \mathrm{P}\right)$ ATP and H2B as a substrate. Kinase products were resolved by SDS-PAGE and transferred to a nitrocellulose membrane. Phospholabeled proteins were visualized by autoradiography. The same membrane was immunoblotted with anti-PKC $\delta$ antibodies as loading control. Results are a representative of three independent experiments

the Hsp27-A mutant (Figure 7a). In contrast, overexpression of Hsp27-D significantly increased the number of apoptotic cells after $9 \mathrm{~h}$ of apigenin treatment, reaching levels similar to the number of apoptotic cells found in cells transfected with the vector control (Figure 7a). Similarly, we found that overexpression of Hsp27-WT or Hsp27-A significantly decreased the percentage of active caspase-3-positive cells at $9 \mathrm{~h}$ (Figure 7b), whereas Hsp27-D overexpressing cells showed a significant increase of apoptosis compared with Hsp27-WT or the Hsp27-A mutant (Figure 7b). Next, lysates from the same cells were used to determine the 


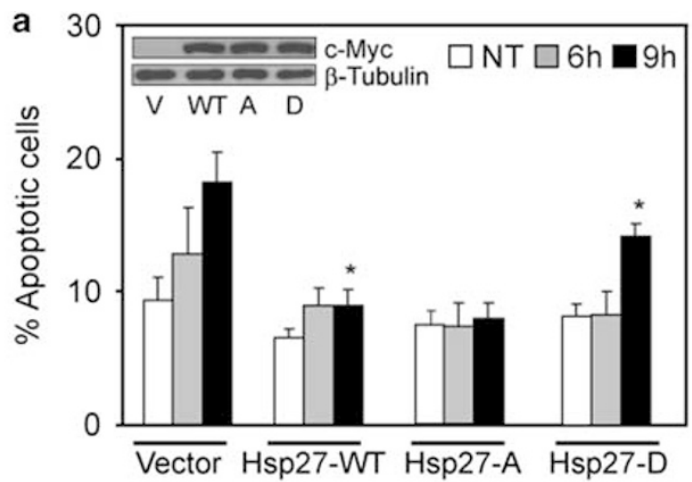

b

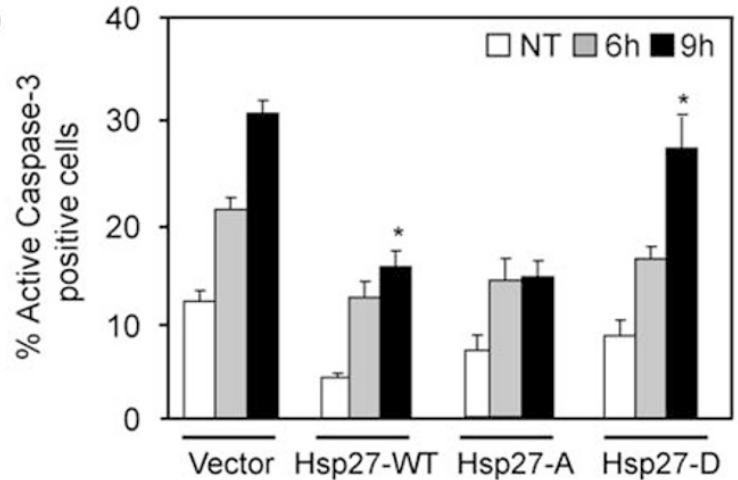

C

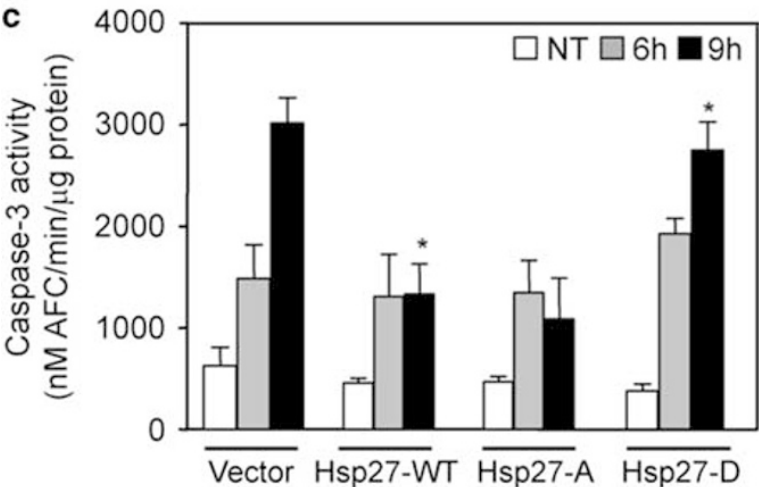

d

Vector Hsp27-WT Hsp27-A Hsp27-D NT 6h 9h NT 6h 9h NT 6h 9h NT 6h 9h

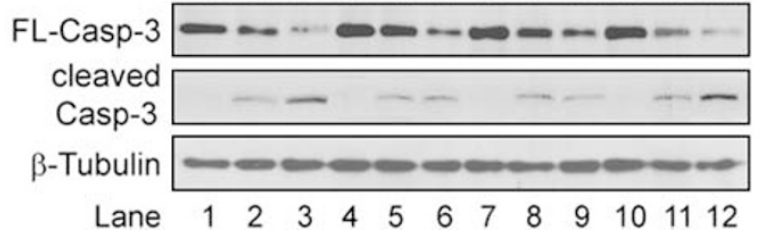

Figure 7 Hsp27 phosphorylation increases apigenin-induced apoptosis. THP-1 cells transiently transfected with empty vector or Hsp27-WT, Hsp27-A, or Hsp27-D were treated with diluent control for $9 \mathrm{~h}$ (NT) or with $50 \mu \mathrm{M}$ apigenin for different times. (a) Percentage of apoptotic cells was determined by Annexin V/7-AAD staining. (b) Active caspase-3 was determined in the same cells by FACS. (c) Lysates from cells obtained in (a) were used to determine caspase-3 activity by the DEVD-AFC assay. (d) Lysates from cells obtained in (a) were used to determine inactive full-length (FL-casp-3) and cleaved caspase-3 (cleaved casp-3) by immunoblotting. Values shown in (a), (b), and (c) represent mean \pm S.E.M., $N=4,{ }^{*} P \leqslant 0.01$

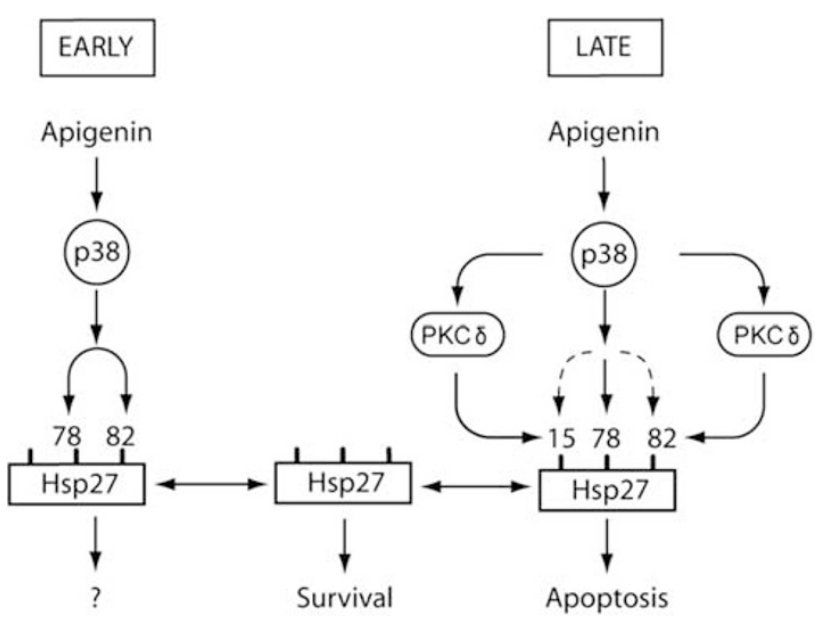

Figure 8 Working model of regulation of Hsp27 phosphorylation during apigenin-induced apoptosis

caspase-3 activity by the DEVD-AFC activity assay. We found that cells transfected with vector control and treated with apigenin for 6 or $9 \mathrm{~h}$ showed increased caspase-3 activity compared with cells treated with diluent (Figure 7c, treated with diluent, referred as NT). Cells expressing Hsp27WT or Hsp27-A have decreased caspase-3 activity at $9 \mathrm{~h}$ compared with cells transfected with the vector control and treated with apigenin for a similar time. In contrast, cells expressing Hsp27-D showed high caspase-3 activity at $9 \mathrm{~h}$, reaching levels of caspase- 3 similar to the cells transfected with the vector control (Figure 7c). Thus, expression of the phosphor-mimic mutant Hsp27-D increases caspase-3 activity. To further assess the effect of the Hsp27 phosphorylation in apoptosis, we evaluated the effect of the Hsp27 phosphor-mimic mutants in the cleavage of caspase3 during the treatment with apigenin. We found that cells expressing Hsp27-WT or Hsp27-A and treated for different times with $50 \mu \mathrm{M}$ apigenin had reduced levels of cleaved caspase-3 and more full-length caspase- 3 after $9 \mathrm{~h}$ of treatment compared with cells transfected with the vector control (Figure 7d). In contrast, cells expressing Hsp27-D showed increased levels of cleaved caspase-3 and reduced levels of full-length caspase- 3 compared with cells expressing either Hsp27-WT or Hsp27-A (Figure 7d). These findings are consistent with the increase in percentage of apoptotic cells, increase in number of cells with active caspase-3, and increase in caspase- 3 activity, obtained by other currently well-accepted methods to determine apoptosis (Figure $7 \mathrm{a}-\mathrm{c}$ ). Collectively, these results suggest that Hsp27 phosphorylation regulates apigenin-induced apoptosis.

\section{Discussion}

Dietary flavonoids are emerging as alternative anticancer agents. Apigenin, a flavonoid abundantly found in fruits and vegetables, induces cell death with variable but relatively low efficacy, in human colon carcinoma, breast cancer, and prostate cancer. $^{6,7}$ Despite its low efficacy in epithelial cells apigenin induces apoptosis with high efficacy in myelogenous 
leukemia cells. ${ }^{8,9}$ However, the mechanisms regulating apigenin-induced apoptosis in leukemia cells remain elusive. Leukemia cells express constitutively high levels of Hsp27, ${ }^{12,15}$ and overexpression of Hsp27 was shown to confer resistance to chemotherapeutic drugs. ${ }^{30}$ Heat or other stresses induce changes in Hsp27 phosphorylation. ${ }^{20,23,31}$ Phosphorylation of Hsp27 was found to affect its oligomerization state by decreasing Hsp27 chaperone activity. ${ }^{20,21,23,32,33}$ In addition, changes in Hsp27 phosphorylation were suggested to modulate Hsp27 cytoprotective activity by probably modulating its interaction with other molecules. ${ }^{22,34}$ Here, we investigated the effect of apigenin in the regulation of Hsp27 and its role in apoptosis.

Our results demonstrated that apigenin had no effect on the total expression level of Hsp27. However, apigenin induced a distinct bimodal phosphorylation of Hsp27, differentially regulated depending on the amino-acid residue, which modulates apigenin-induced apoptosis. Under stress conditions and heat shock, Hsp27 is phosphorylated in vivo on S15, $\mathrm{S} 78$, and $\mathrm{S} 82$ through a signal kinase cascade regulated by p38 and $\operatorname{PKC} \delta .{ }^{20,23,26}$ We found that apigenin induced unique bimodal kinetics of Hsp27 phosphorylation (Figure 8). An 'early' phosphorylation of Hsp27 after 15 min of treatment with apigenin was only observed on S78 and S82 and was regulated by the p38-MAPK (Figures 1 and 2). This 'early' phosphorylation of Hsp27 precedes the activation of caspase-3 and ROS production, two events we previously reported, and further studies will need to be conducted to investigate the role of the 'early' phosphorylation. Using pharmacological inhibitors and silencing experiments, we found that Hsp27 is phosphorylated on the three residues, $\mathrm{S} 15, \mathrm{~S} 78$, and S82, after treatment with apigenin for $6 \mathrm{~h}$ (Figures 1, 4 and 5). We referred to this phosphorylation as 'late' phosphorylation (Figure 8). Notably, these results indicate a different signaling network that seems to regulate the phosphorylation of these three amino-acid residues. Phosphorylation of S78 was p38 dependent, whereas phosphorylation of $\mathrm{S} 15$ and $\mathrm{S} 82$ was regulated by $\mathrm{p} 38$ and PKC $\delta$ (Figure 8). We cannot rule out the possibility that p38 could regulate $\mathrm{S} 15$ and $\mathrm{S} 82$ phosphorylation but with a competitive disadvantage to that of $\operatorname{PKC} \delta$ and, if so, only regulate the phosphorylation of these residues directly in the absence of $\mathrm{PKC} \delta$ activity (Figure 8). Unlike Hsp27 phosphorylation induced by oxidative stress, ${ }^{35}$ we found that the phosphorylation of Hsp27 induced by apigenin was independent of ERK (Figure 3). During heat and metabolic stresses, the main Hsp27 sites phosphorylated in vivo were S78 and S82. ${ }^{24}$ Findings by Gaestel and coworkers, however, reported that incorporation on S15, while 10 times lower, was also observed upon stimulation. ${ }^{23}$ Phosphorylation of Hsp27 is catalyzed directly by the MKK2/3 through p38 activation during different stress conditions. ${ }^{34}$ Upon activation of $\mathrm{p} 38$, Hsp25, the Hsp27 mouse homolog, can also be phosphorylated by $\mathrm{PKC} \delta .^{26}$ Unlike radiation-induced Hsp27 phosphorylation, where $\mathrm{PKC} \delta$ is upstream of $\mathrm{p} 38,{ }^{36}$ we found that the activation of $\mathrm{PKC} \delta$ induced by apigenin was mediated by $\mathrm{p} 38$ (Figures 6 and 7).

The bimodal Hsp27 phosphorylation kinetics observed in leukemia cells treated with apigenin is so far unique and suggests a complex regulatory network responsible of
Hsp27 regulation (Figure 8). Although the biological effect of the 'early' Hsp27 phosphorylation will need further studies, we found that the 'late' phosphorylation of Hsp27 regulates apigenin-induced apoptosis. The expression of phosphormimic Hsp27-D mutant was shown previously to regulate Hsp27 oligomerization state but failed to protect cells against oxidative stress. ${ }^{23} \mathrm{We}$ found that overexpression of the same phosphor-mimic mutant Hsp27-D rendered leukemia cells more susceptible to apigenin-induced apoptosis by increasing the percentage of apoptotic cells after $9 \mathrm{~h}$, as determined by AnnexinV/7-AAD, active caspase-3 staining, caspase-3 activity, and the proteolytic cleavage of caspase-3 (Figure 7).

Collectively, our studies identify a complex signaling kinase network involved in Hsp27 phosphorylation induced by apigenin and provide novel insights into the molecular mechanism responsible for the anticancer activity of this flavonoid.

\section{Materials and Methods}

Cell culture and reagents. THP-1 cells were obtained from the ATCC and cultured in RPMI 1640 medium with L-glutamine (BioWhittaker, Walkersville, MD, USA) supplemented with 5\% FBS (Atlas Biological, Lawrenceville, GA, USA), 1\% penicillin-streptomycin (Lonza, Walkersville, MD, USA) and at a density lower than $0.5 \times 10^{6} \mathrm{cell} / \mathrm{s} / \mathrm{ml}$. All cells were grown at $37^{\circ} \mathrm{C}$ in a humidified atmosphere of $95 \%$ air and $5 \% \mathrm{CO}_{2}$. Apigenin (A-Apin Chemicals, Oxon, UK), the PKC $\delta$ inhibitor rottlerin and the diluent dimethyl sulfoxide (DMSO) were obtained from SigmaAldrich (St Louis, MO, USA). In all the experiments, cells were treated with $50 \mu \mathrm{M}$ apigenin. The p38 inhibitor SB203580, the ERK inhibitor PD98059 (Calbiochem, San Diego, CA, USA), and rottlerin were dissolved in DMSO and added to the cell culture $1 \mathrm{~h}$ before the addition of apigenin at 10,25 , and $15 \mu \mathrm{M}$, respectively.

Extract preparation and western blot analysis. Cells were collected by centrifugation, washed with PBS and lysed for $1 \mathrm{~h}$ at $4{ }^{\circ} \mathrm{C}$ with continuous vortexing in ice-cold buffer $B$ (50 mM HEPES pH 7.4, 2.5 mM EGTA, 1 mM EDTA, $150 \mathrm{mM} \mathrm{NaCl}, 10 \%$ glycerol, $0.1 \%$ Tween-20 containing the phosphatase inhibitors: $20 \mathrm{mM} \mathrm{NaF}, 10 \mathrm{mM}$ Na glycerophosphate, $5 \mathrm{mM}$ Na pyrophosphate, $1 \mathrm{mM}$ orthovanadate, $1 \mathrm{mM}$ DTT, $0.1 \mathrm{mM}$ PMSF, $2 \mu \mathrm{g} / \mathrm{ml}$ of protease inhibitors: chymostatin, pepstatin, leupeptin, antipain). Extracts were then centrifuged for $5 \mathrm{~min}$ at 14000 r.p.m. at $4^{\circ} \mathrm{C}$. Laemmli buffer containing $2.5 \% \beta$-mercaptoethanol (BioRad, Hercules, CA, USA) was added directly to the cell lysates, and the samples were boiled for $5 \mathrm{~min}$ before loading onto gels. Equal amounts of protein were loaded and separated by SDS-PAGE, transferred onto nitrocellulose membranes, and probed with antibodies of interest followed by enhanced chemiluminescence with secondary antibodies conjugated to horseradish peroxidase (Amersham Biosciences, Arlington Heights, IL, USA). Phospho-Hsp27-pS15 (SPA-525), phospho-Hsp27-pS78 (SPA-523), phospho-Hsp27-pS82 (SPA-524), and total Hsp27 (SPA-803) antibodies were obtained from Stressgen (British Columbia, Canada). Antibodies used for MAPK, total p44/42 (referred as ERK in the text, cat. 9102), phospho-ERK (9101), phospho-p38 (9211), and total p38 (9212) were from Cell Signaling (Boston, MA, USA). PKC $\delta$ (SC-937) antibody was obtained from Santa Cruz Biotechnology (Santa Cruz, CA, USA), anticleaved active caspase-3 antibodies (9661S, Clone Asp 175) were from Cell Signaling (Danvers, MA, USA) anti-full-length inactive caspase-3 antibodies (610323) were from BD Biosciences (San Jose, CA, USA) and $\beta$-Tubulin (05-661) was acquired from Millipore (Billerica, MA, USA). Densitometry analysis was performed using Quantity One software (BioRad), and data were normalized using the loading control $\beta$-Tubulin. For Figure 4 , the data representing the densitometry analysis obtained from the amount of phospho-Hsp27 divided by the total amount of Hsp27 was represented as a percentage of the amount found in non-treated cells.

Transient transfection, siRNA, and flow cytometry. THP-1 cells were transiently transfected using the Amaxa Nucleofector (Amaxa, Cologne, Germany) program V-001 at a final concentration of $1 \times 10^{7} \mathrm{cell} / \mathrm{s} / \mathrm{ml}$ in a $100 \mu \mathrm{l}$ volume, as previously described. ${ }^{15}$ Five micrograms of empty vector (pcDNA35myc), vector containing Hsp27-WT, a Hsp27-phosphor-mutant in which S15, S78, and $\mathrm{S} 82$ were replaced by Asp and is referred in the text as Hsp27-D or in which $\mathrm{S} 15, \mathrm{~S} 78$, and S82 were replaced by Ala and is referred as Hsp27-A (a generous gift 
from Dr. Gaestele ${ }^{23}$ ) were used for transfections. The transfection efficiency for the clones used, Hsp27-WT, Hsp27-D, and Hsp27-A, was similar and averaged 50\% as determined by FACS analysis in cells stained with anti-Myc antibodies (not shown), and is similar to the transfection efficiency previously reported by this method. ${ }^{15}$ Twenty-four hours after transfection, the cells were treated with $50 \mu \mathrm{M}$ apigenin for the indicated times. For silencing experiments, THP-1 cells were transfected with $50 \mathrm{nM}$ siRNA-p38 (Signal Silencing pool p38-MAPK siRNA; Cell Signaling, Cat: 6386), siRNA scramble control (Qiagen, Valencia, CA, USA; Cat: 1027284 ) or $100 \mathrm{nM}$ siRNA-PKC $\delta$ (Qiagen, Cat: 1027283 using the Amaxa nucleofector program V-001 as previously described. ${ }^{9}$ Cells were treated, as described in the text, $48 \mathrm{~h}$ after siRNA transfections. Apoptosis was assessed by staining cells with AnnexinV/7-AAD using the Annexin V-FITC apoptosis detection kit, following the manufacturer's specification (BD Biosciences). Alternatively, cells were washed with PBS and resuspended at a concentration of $2 \times 10^{6} \mathrm{cell} / \mathrm{s} / \mathrm{ml}$ in blocking buffer (PBS containing 1\% FBS and $200 \mu \mathrm{g} / \mathrm{ml}$ human total $\mathrm{lgG}$ ) and incubated for $30 \mathrm{~min}$ on ice, washed twice with blocking buffer, resuspended in $250 \mu \mathrm{l} \mathrm{cytofix/cytoperm,} \mathrm{and} \mathrm{incubated} \mathrm{for} 20 \mathrm{~min}$ on ice with $20 \mu \mathrm{l}$ of FITCconjugated antiactive-caspase-3 antibodies (BD Pharmingen, San Jose, CA, USA). Cells were rinsed twice with Perm/Wash buffer and resuspended in blocking buffer for flow analysis. Flow cytometry analysis was performed using Becton Dickinson FACS Calibur (San Jose, CA, USA) using Cell Quest version 3.3 software as previously described. ${ }^{15}$

Immunoprecipitations and in vitro kinase assays. Fifty micrograms of THP-1 lysates were immunoprecipitated overnight at $4^{\circ} \mathrm{C}$ with $200 \mathrm{ng}$ of anti-PKC $\delta$ antibody (SC-937) or IgG isotype as control (SC-2027), followed by $1 \mathrm{~h}$ incubation with $25 \mu \mathrm{l}$ of protein G-agarose beads (Amersham Biosciences). Immunoprecipitates were rinsed three times with buffer $B$ and one time with kinase buffer (25 mM HEPES pH 7.4, $10 \mathrm{mM} \mathrm{MnCl}_{2}, 1 \mathrm{mM} \mathrm{MgCl}_{2}, 1 \mathrm{mM} \mathrm{DTT}, 0.1 \mathrm{mM}$ PMSF) and subjected to in vitro kinase assays by incubating the beads for $1 \mathrm{~h}$ at $37^{\circ} \mathrm{C}$ in the presence of $40 \mu$ l of kinase assay buffer containing $2 \mu \mathrm{Ci}$ of $\left(\gamma^{32} \mathrm{P}\right)$ ATP (Perkin Elmer, Boston, MA, USA), $0.5 \mathrm{mM}$ ATP, $200 \mu \mathrm{g} / \mathrm{ml}$ phosphatidyl-serine, and $20 \mu \mathrm{g} / \mathrm{ml}$ diacylglycerol, in the presence of $1 \mu \mathrm{g} \mathrm{H} 2 \mathrm{~B}$ (Roche Applied Science, Mannheim, Germany) as exogenous substrate. All reactions were stopped by the addition of Laemmli buffer, boiled for $5 \mathrm{~min}$ and loaded onto SDS-PAGE.

Caspase-3 activity assay. Active caspase- 3 was determined by the AFC assay, as previously described. ${ }^{37}$ Lysates were incubated in a cyto-buffer $(10 \%$ glycerol, $50 \mathrm{mM}$ Pipes, pH 7.0, $1 \mathrm{mM}$ EDTA, containing $1 \mathrm{mM}$ DTT) containing $20 \mu \mathrm{M}$ of the tetrapeptide substrate DEVD-AFC. The tetrapeptide was obtained from Enzyme Systems Products (Livermore, CA, USA). Release of free AFC was determined using a Cytofluor 4000 fluorometer (Perseptive Company, Framingham, MA, USA; Filters: excitation; $400 \mathrm{~nm}$, emission; $508 \mathrm{~nm}$ ).

Statistical analysis. All data are expressed as mean \pm S.E.M., and two way Student's $t$-test comparisons were used to assess statistical significance. Statistical significance is stated in the text.

\section{Conflict of interest}

The authors declare no conflict of interest.

Acknowledgements. We thank $\operatorname{Dr}$ Gaestel for generously providing the Hsp27 clones. We thank Dr Eubank for assistance with graphics. This work was supported by Grants R01HL075040-01 and NSF-MCB-0542244 to AID.

1. Di Carlo G, Mascolo N, Izzo AA, Capasso F. Flavonoids: old and new aspects of a class of natural therapeutic drugs. Life Sci 1999; 65: 337-353.

2. Middleton E, Kandaswami C, Theoharides TC. The effects of plant flavonoids on mammalian cells: implications for inflammation, heart disease, and cancer. Pharmacol Rev 2000; 43: 673-751.

3. Havsteen $\mathrm{BH}$. The biochemistry and medical significance of the flavonoids. Pharmacol Ther 2002; 96: 67-202.

4. Rice-Evans $\mathrm{C}$, Spencer JP, Schroeter $\mathrm{H}$, Rechner AR. Bioavailability of flavonoids and potential bioactive forms in vivo. Drug Metabol Drug Interac 2000; 17: 291-310.

5. Stafford HA. Flavonoid Metabolism. CRC Press, Inc.: Boca Raton, USA, 1990.

6. Way TD, Kao MC, Lin JK. Apigenin induces apoptosis through proteasomal degradation of HER2/neu in HER2/neu-overexpressing breast cancer cells via the phosphatidylinositol 3-kinase/Akt-dependent pathway. J Biol Chem 2004; 279: 4479-4489.

7. Wang W, Heideman L, Chung CS, Pelling JC, Koehler KJ, Birt DF. Cell-cycle arrest at $\mathrm{G} 2 / \mathrm{M}$ and growth inhibition by apigenin in human colon carcinoma cell lines. Mol Carcinog 2000; 28: 102-110.

8. Wang IK, Lin-Shiau SY, Lin JK. Induction of apoptosis by apigenin and related flavonoids through cytochrome $c$ release and activation of caspase- 9 and caspase-3 in leukaemia HL-60 cells. Eur J Cancer 1999; 35: 1517-1525.

9. Vargo MA, Voss OH, Poustka F, Cardounel AJ, Grotewold E, Doseff Al. Apigenin-inducedapoptosis is mediated by the activation of PKC delta and caspases in leukemia cells. Biochem Pharmacol 2006; 72: 681-692.

10. Ruela-de-Sousa RR, Fuhler GM, Blom N, Ferreira CV, Aoyama H, Peppelenbosch MP. Cytotoxicity of apigenin on leukemia cell lines: implications for prevention and therapy. Cell Death Disease 2010; 1: 1-12.

11. Hartl FU, Hayer-Hartl M. Molecular chaperones in the cytosol: from nascent chain to folded protein. Science (New York, NY) 2002; 295: 1852-1858.

12. Lanneau D, Brunet M, Frisan E, Solary E, Fontenay M, Garrido C. Heat shock proteins: essential proteins for apoptosis regulation. J Cel Mol Med 2008; 12: 743-761.

13. Charette SJ, Lavoie JN, Lambert H, Landry J. Inhibition of Daxx-mediated apoptosis by heat shock protein 27. Mol Cel Biol 2000; 20: 7602-7612.

14. Bruey J, Ducasse C, Bonniaud P, Ravagnan L, Susin S, Diaz-Latoud C et al. Hsp27 negatively regulates cell death by interacting with cytochrome $c$. Nat Cell Biol 2000; 2: 645-652.

15. Voss OH, Batra S, Kolattukudy SJ, Gonzalez-Mejia ME, Smith JB, Doseff Al. Binding of caspase-3 prodomain to heat shock protein 27 regulates monocyte apoptosis by inhibiting caspase-3 proteolytic activation. J Biol Chem 2007; 282: 25088-25099.

16. Hansen RK, Parra I, Lemieux P, Oesterreich S, Hilsenbeck SG, Fuqua SAW. Hsp27 overexpression inhibits doxorubicin-induced apoptosis in human breast cancer cells. Breast Cancer Res Treat 1999; 56: 187-196.

17. Thomas X, Campos L, Mounier C, Cornillon J, Flandrin P, Le QH et al. Expression of heatshock proteins is associated with major adverse prognostic factors in acute myeloid leukemia. Leukemia Res 2005; 29: 1049-1058.

18. de Graauw M, Tijdens I, Cramer R, Corless S, Timms JF, van de Water B. Heat shock protein 27 is the major differentially phosphorylated protein involved in renal epithelial cellular stress response and controls focal adhesion organization and apoptosis. J Biol Chem 2005; 280: 29885-29898.

19. Geum D, Son GH, Kim K. Phosphorylation-dependent cellular localization and thermoprotective role of heat shock protein 25 in hippocampal progenitor cells. J Biol Chem 2002; 277: 19913-29921.

20. Nozaki J, Takehana M, Kobayashi S. UVB irradiation induces changes in cellular localization and phosphorylation of mouse HSP27. Photochem Photobiol 1997; 65: 843-848.

21. Kato K, Hasegawa K, Goto S, Inaguma Y. Dissociation as a result of phosphorylation of an aggregated form of the small stress protein, hsp27. J Biol Chem 1994; 269: 11274-11278.

22. Benndorf R, Hayess K, Ryazantsev S, Wieske M, Behlke J, Lutsch G. Phosphorylation and supramolecular organization of murine small heat shock protein HSP25 abolish its actin polymerization-inhibiting activity. J Biol Chem 1994; 269: 20780-20784.

23. Rogalla T, Ehrnsperger M, Preville X, Kotlyarov A, Lutsch G, Ducasse $C$ et al. Regulation of Hsp27 oligomerization, chaperone function, and protective activity against oxidative stress/ tumor necrosis factor alpha by phosphorylation. J Biol Chem 1999; 274: 18947-18956.

24. Landry J, Lambert H, Zhou M, Lavoie JN, Hickey E, Weber LA et al. Human HSP27 is phosphorylated at serines 78 and 82 by heat shock and mitogen-activated kinases that recognize the same amino acid motif as S6 kinase II. J Biol Chem 1992; 267: 794-803.

25. Stokoe D, Engel K, Campbell DG, Cohen P, Gaestel M. Identification of MAPKAP kinase 2 as a major enzyme responsible for the phosphorylation of the small mammalian heat shock proteins. FEBS Lett 1992; 313: 307-313.

26. Lee Y-L, Lee D-H, Cho C-K, Bae S, Jhon G-J, Lee S-J et al. Hsp25 inhibits protein kinase C delta-mediated cell death through direct interaction. J Biol Chem 2005; 280: 18108-18119.

27. Maizels ET, Peters C, Kline M, Cutler R, Shanmugam M, Hunzicker-Dunn M. Heat-shock protein-25/27 phosphorylation by the delta isoform of protein kinase C. Biochem J 1998; 332: 703-712.

28. Coxon PY, Rane MJ, Uriarte S, Powell DW, Singh S, Butt W et al. MAPK-activated protein kinase-2 participates in p38 MAPK-dependent and ERK-dependent functions in human neutrophils. Cell Signal 2003; 15: 993-1001.

29. Denhardt DT. Signal-transducing protein phosphorylation cascades mediated by Ras/Rho proteins in the mammalian cell: the potential for multiplex signalling. Biochem J 1996; 318 (Part 3): 729-747.

30. Schmitt E, Gehrmann M, Brunet M, Multhoff G, Garrido C. Intracellular and extracellular functions of heat shock proteins: repercussions in cancer therapy. J Leuk Biol 2007; 81: 15-27.

31. Mehlen P, Arrigo A-P. The serum-induced phosphorylation of mammalian hsp correlates with changes in its intracellular localization and levels of oligomerization. Eur J Biochem 1994; 221: 327-334.

32. Mehlen P, Remy-Kretz C, Briolay J, Fostan P, Mirault M-E, Arrigo A-P. Intracellular reactive oxygen species as apparent modulators of heat shock protein 27 (hsp27) structural organization and phosphorylation in basla and tumor necrosis factor alpha-treated T47D human carcinoma cells. Biochem J 1995; 312: 367-375.

33. Vertii A, Hakim C, Kotlyarov A, Gaestel M. Analysis of properties of small heat shock protein Hsp25 in MAPK-activated protein kinase 2 (MK2)-deficient cells: MK2-dependent 
insolubilization of Hsp25 oligomers correlates with susceptibility to stress. J Biol Chem 2006; 281: 26966-26975.

34. Garrido C. Size matters: of the small HSP27 and its large oligomers. Cell Death Differ 2002; 9: 483-485.

35. Lee Y-J, Cho H-N, Jeoung D-I, Soh J-W, Cho C-K, Bae S et al. Hsp25 overexpression attenuates oxidative stress-induced apoptosis: roles of ERK $1 / 2$ signaling and manganese superoxide dismutase. Free Radical Biol Med 2004; 36: 429-444.

36. Lee YJ, Lee D-H, Cho C-K, Chung H-Y, Bae S, Jhon G-J et al. Hsp25 inhibits radiationinduced apoptosis through reduction of PKC delta-ROS mediated production. Oncogene 2005; 24: 3715-3725.
37. Voss $\mathrm{OH}$, Kim S, Wewers MD, Doseff Al. Regulation of monocyte apoptosis by the protein kinase $C$ delta-dependent phosphorylation of caspase-3. J Biol Chem 2005; 280 : $17371-17379$

Cell Death and Disease is an open-access journal published by Nature Publishing Group. This work is licensed under the Creative Commons Attribution-Noncommercial-No Derivative Works 3.0 Unported License. To view a copy of this license, visit http://creativecommons.org/licenses/by-nc-nd/3.0/ 